\title{
Probing Neutrino Hierarchy and Chirality via Wakes
}

\author{
Hong-Ming Zhu, ${ }^{1,2}$ Ue-Li Pen, ${ }^{3,4}$ Xuelei Chen, ${ }^{1,2,5}$ and Derek Inman ${ }^{3}$ \\ ${ }^{1}$ Key Laboratory for Computational Astrophysics, National Astronomical Observatories, \\ Chinese Academy of Sciences, 20A Datun Road, Beijing 100012, China \\ ${ }^{2}$ University of Chinese Academy of Sciences, Beijing 100049, China \\ ${ }^{3}$ Canadian Institute for Theoretical Astrophysics, 60 St. George Street, Toronto, Ontario M5S 3H8, Canada \\ ${ }^{4}$ Canadian Institute for Advanced Research, CIFAR Program in Gravitation and Cosmology, Toronto, Ontario M5G 1Z8, Canada \\ ${ }^{5}$ Center of High Energy Physics, Peking University, Beijing 100871, China
}

(Dated: April 6, 2018)

\begin{abstract}
The relic neutrinos are expected to acquire a bulk relative velocity with respect to the dark matter at low redshifts, and neutrino wakes are expected to develop downstream of the dark matter halos. We propose a method of measuring the neutrino mass based on this mechanism. This neutrino wake will cause a dipole distortion of the galaxy-galaxy lensing pattern. This effect could be detected by combining upcoming lensing surveys with a low redshift galaxy survey or a $21 \mathrm{~cm}$ intensity mapping survey, which can map the neutrino flow field. The data obtained with LSST and Euclid should enable us to make a positive detection if the three neutrino masses are quasidegenerate with each neutrino mass of $\sim 0.1 \mathrm{eV}$, and a future high precision $21 \mathrm{~cm}$ lensing survey would allow the normal hierarchy and inverted hierarchy cases to be distinguished, and even the right-handed Dirac neutrinos may be detectable.
\end{abstract}

PACS numbers: 98.62.Sb, 14.60.Pq, 95.35.+d, 95.80.+p

Introduction.-The squared mass differences of the three neutrino species have been measured from neutrino oscillation experiments, but the individual masses are still unknown. Based on the measured mass square differences, the neutrino masses may form the so called normal hierarchy $\left(m_{1} \sim\right.$ $\left.m_{2} \ll m_{3}\right)$ or the inverted hierarchy $\left(m_{3} \ll m_{1} \sim m_{2}\right)$, or they may be quasidegenerate $\left(m_{1} \sim m_{2} \sim m_{3}\right)\lfloor 1]$. Determining the neutrino mass hierarchy is a very important problem in modern physics [2]. The suppression of the matter power spectrum on small scales provides a way to measure the sum of neutrino masses [3, 4], complementary to particle physics experiments, and in some cases the combination of these two approaches may allow the determination of the mass hierarchy [5].

Recently, it was shown that a bulk relative velocity field between neutrinos and cold dark matter (CDM) exists, with coherent flows over several Mpc [6]. This produces a crosscorrelation dipole between CDM and neutrinos on large scales. While on nonlinear scales, as neutrinos flow over dark matter halos, they are gravitationally focused into a wake. This downstream excess can be observed through gravitational lensing. This wake is unique to neutrinos as the CDMbaryon relative velocity is far too small to mimic the effect. The full three-dimensional relative velocity field can be computed from the galaxy density field using linear perturbation theory with good accuracy. By exploiting the asymmetry of the relative velocity, the neutrino wakes can be isolated and used to determine the neutrino masses.

Besides the neutrino masses, the very nature of the neutrinos, i.e., whether they are Majorana or Dirac particles, is still unknown. If neutrinos are Majorana particles, the lepton number conservation law is broken, and this may induce neutrinoless $\beta$ decay, but so far such decays have not been detected despite many search efforts [7]. This question may be answered if the big bang relic neutrinos can be detected with a tritium capture experiment [8], for the total capture rate in the Majorana case could be twice as large as that of the Dirac case [9]. However, detecting the relic neutrinos is very difficult due to their small kinetic energy, and furthermore, even if the relic neutrinos are detected, this effect is confounded by the fact that the local neutrino overdensity due to gravitational clustering is unknown. Here, we note that for Dirac neutrinos there are right-handed neutrinos $\left(\nu_{R}\right.$ 's) distinct from the left-handed ones $\left(\nu_{L}\right.$ 's), and they could have been produced during the big bang. In many beyond the standard model theories, e.g., the models with a heavier right-handed coupling gauge boson $Z^{\prime}$ [10], the left-right symmetry is restored at high energy, then in the very early Universe there would be a thermalized $\nu_{R}$ background in addition to the $\nu_{L}$ one. However, the left-right symmetry must be broken at low energy, and as the $Z^{\prime}$ is heavier than the $Z$ boson, the primordial $\nu_{R}$ 's would decouple before the $\nu_{L}$ 's, and evolve as a separate relic background. At a later time, these neutrinos would propagate in mass eigenstates, with both right and left (regenerated from Yukawa coupling) components, but their temperature and number density would be distinct from the primordial $\nu_{L}$ background, which decoupled much later. Since the relative velocity with respect to CDM depends on the initial velocity dispersion [6], the relative velocity field of the $\nu_{R}$ including its magnitude and direction would also be different, thus enabling a new way to answer this important question. (In the seesaw models of neutrino masses [11], the light $\nu_{L}$ 's are primarily Majorana particles. There are also right-handed neutrinos in such models, but they are very heavy and should have decayed in the early Universe.) In this Letter we discuss how these neutrino wakes are produced by nonlinear CDM halos and compute the expected lensing signals, and investigate the observability with upcoming surveys. Moreover, we also delineate the evolution of the $\nu_{R}$ 's for the case of Dirac neutrinos, and consider the detectability with this method. 
Neutrino wakes and lensing signal.-Because of the free streaming of neutrinos over large scales, the bulk flow of neutrinos grows slowly compared to that of CDM; as a result a bulk relative velocity field between neutrinos and CDM is induced. The relative velocity field can be computed from the primordial density perturbations and its variance is given by

$$
\left\langle v_{\nu c}^{2}\right\rangle(z)=\int \frac{d k}{k} \Delta_{\zeta}^{2}(k)\left[\frac{\theta_{\nu}(k, z)-\theta_{c}(k, z)}{k}\right]^{2},
$$

where $\theta \equiv \nabla \cdot \boldsymbol{v}$ is the velocity divergence transfer function and $\Delta_{\zeta}^{2}(k)$ is the primordial curvature power spectrum [6]. This variance was computed for the $\nu_{L}$ in Ref. [6] where it was found to be comparable with the thermal velocity at late times. The $\nu_{L}$ relative velocity power spectrum is shown in Fig. 1 for the case of a single $0.05 \mathrm{eV}$ neutrino. The velocity coherent scale, which is defined as the distance where the relative velocity correlation function drops to half its maximum value [6], is $R=14.5 \mathrm{Mpc} / h$ for the $\nu_{L}$ at $z=0.3$. In the following calculations, we take $\sqrt{\left\langle v_{\nu_{L} c}^{2}\right\rangle}=418 \mathrm{~km} / \mathrm{s}$ at $z=0.3$ as the typical relative velocity. The one-dimensional neutrino velocity dispersion is $\sigma(z)=2.077 T_{\nu}(z) / m_{\nu} \simeq 2716 \mathrm{~km} / \mathrm{s}$ for the $\nu_{L}$ at $z=0.3$.

We now compute the neutrino wake induced by a dark matter halo of mass $M$ via linear response theory. We approximate the neutrinos' distribution as Maxwell Boltzmann, which is sufficiently accurate for the relevant densities and temperatures. Here, we take the halo as the origin point. Assuming neutrinos flow over the dark matter halo coherently with a velocity $v_{\nu c}(z)=\sqrt{\left\langle v_{\nu_{1} c}^{2}\right\rangle}(z)$, the neutrino density contrast at point $\boldsymbol{r}$ is given by [12]

$$
\begin{aligned}
\delta_{\nu}(\boldsymbol{r}) & =\frac{r_{B}}{r} \exp \left(-\frac{v_{\nu c}^{2} \sin ^{2} \vartheta}{2 \sigma^{2}}\right)\left[1+\operatorname{erf}\left(\frac{v_{\nu c} \cos \vartheta}{\sqrt{2} \sigma}\right)\right] \\
& \approx \frac{r_{B}}{r}\left(1+\frac{2}{\sqrt{\pi}} X \cos \vartheta-X^{2} \sin ^{2} \vartheta\right)
\end{aligned}
$$

where $r_{B}(z)=G M / \sigma^{2}(z)$ is the Bondi radius, $\vartheta$ is the angle between the relative velocity and $\boldsymbol{r}$, and $X(z)=$ $v_{\nu c}(z) /[\sqrt{2} \sigma(z)]$. The approximation in the second line is better than $95 \%$ for the cases we consider.

With Eq. (2) in hand we can compute the expected lensing signal from this wake. The geometry is shown in Fig. 2. Taking the $z$ axis as the direction of our line of sight (l.o.s.) and the relative velocity $\boldsymbol{v}_{\nu c}$ to lie in the $x-z$ plane at an angle $\theta$ from the $z$ axis, then the induced density contrast at any point $\boldsymbol{r} \equiv(x, y, z)$ can be determined from Eq. (2) via coordinate transformations. Here, we also define the polar coordinates $(\varpi, \phi)$ on the lens $(x-y)$ plane, $x=\varpi \cos \phi, y=\varpi \sin \phi$, for later use.

The perturbed surface neutrino mass density is obtained by integration along the 1.o.s.: $\Sigma_{\nu}(x, y)=$ $\rho_{0} \int_{-a L / 2}^{a L / 2} d z \delta_{\nu}(x, y, z)$, where $\rho_{0}$ is the unperturbed neutrino mass density, $a$ is the scale factor, and $L$ is the effective coherent scale of the neutrino-CDM relative flow field, defined as $(4 / 3) \pi R^{3}=L^{3}$. We find $L=23.4 \mathrm{Mpc} / h$ for the $\nu_{L}$ at

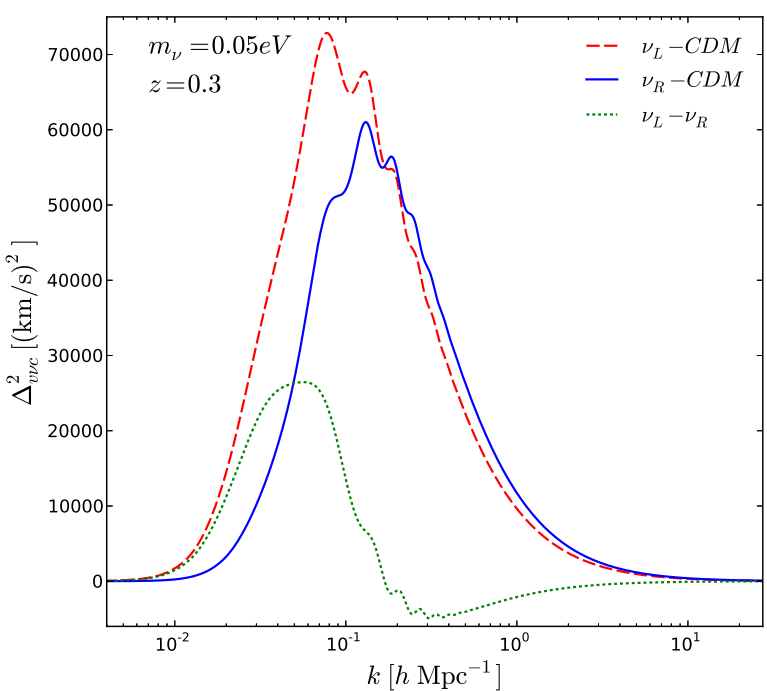

FIG. 1: Relative velocity power spectra between the $\nu_{L}, \nu_{R}$, and $\mathrm{CDM}$ at $z=0.3$.

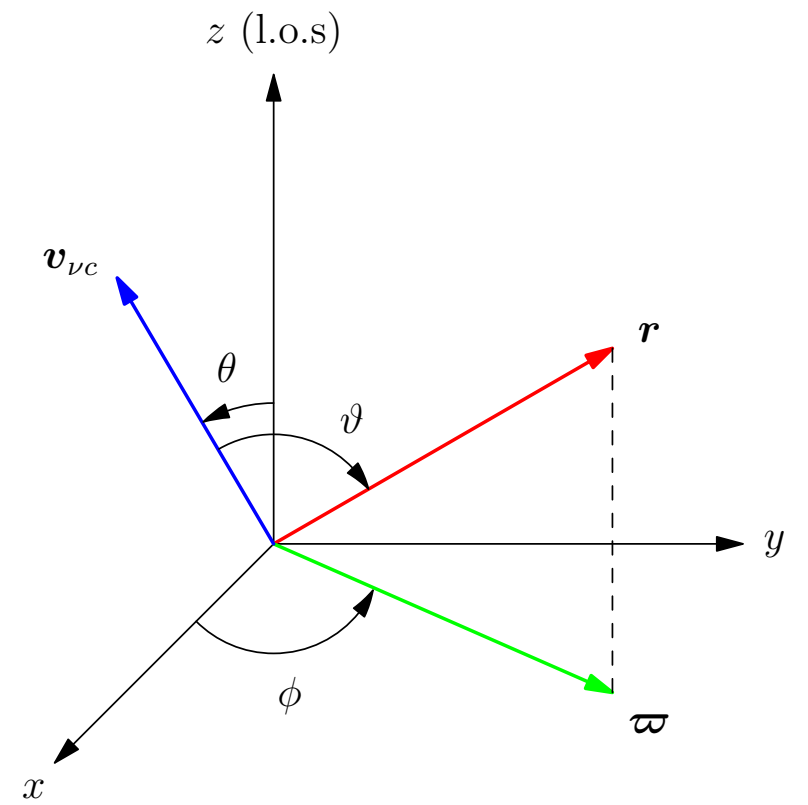

FIG. 2: The coordinate system employed in calculating the lensing signal. $\boldsymbol{v}_{\nu c}$ is the relative velocity, $\boldsymbol{r}$ is the point at which we compute the density contrast, and $(\varpi, \phi)$ are polar coordinates in the lensing plane.

$z=0.3$ for $m_{\nu}=0.05 \mathrm{eV}$. We first consider the contribution from a single halo at $z=0.3$. Using Eq. (2), we obtain

$$
\begin{aligned}
& \frac{\Sigma_{\nu}}{\rho_{0} r_{B}}=\left(1-X^{2} \sin ^{2} \theta\right) \ln \left[\frac{1+\sin \eta}{1-\sin \eta}\right]+\frac{4 \eta X}{\sqrt{\pi}} \sin \theta \cos \phi \\
& +X^{2}\left(3 \sin ^{2} \theta-2\right) \sin \eta-X^{2}\left(\cos ^{2} \theta-1\right) \sin \eta \cos 2 \phi,
\end{aligned}
$$

where $\eta=\arctan (a L / 2 \varpi)$. The contribution of neutrinos in a redshift slice to the weak lensing convergence is given by $\kappa(\varpi, \phi)=\Sigma_{\nu}(\varpi, \phi) \omega(\chi)$, where $\omega(\chi)=$ 


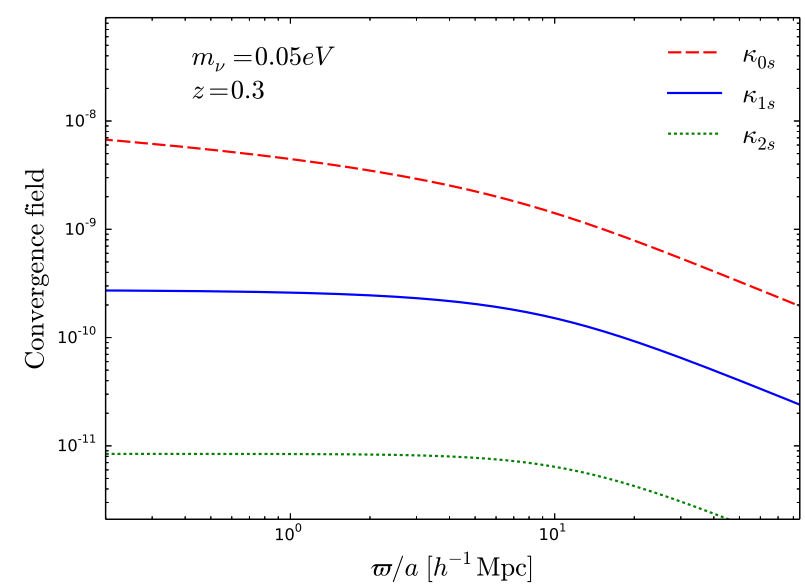

FIG. 3: Different components of the convergence field for a single $10^{13} M_{\odot}$ halo at $z=0.3$, with $m_{\nu}=0.05 \mathrm{eV}$ when $\theta=\pi / 2$. $a=1 /(1+z)$ is the scale factor and $\varpi / a$ shows the comoving distance at redshift $z=0.3$.

$4 \pi \mathrm{Ga} \chi \int_{z}^{\infty} d z_{s} n\left(z_{s}\right)\left(1-\chi / \chi_{s}\right)$ is the lensing weight, $\chi$ and $\chi_{s}$ are comoving distances to the halo and the source, and $n\left(z_{s}\right)$ is the redshift distribution of the sources normalized to unity. We adopt the source distribution characterized by $n\left(z_{s}\right) \propto z_{s}^{\alpha} \exp \left[-\left(z_{s} / z^{*}\right)^{\beta}\right]$ with $\alpha=2, z^{*}=0.5$, and $\beta=1$, which corresponds to the Large Synoptic Survey Telescope (LSST) survey [13]. The lensing weight peaks at $z=0.38$. The convergence can be arranged into several multipole terms:

$$
\kappa(\varpi, \phi)=\kappa_{0}(\varpi)+\kappa_{1}(\varpi) \cos \phi+\kappa_{2}(\varpi) \cos 2 \phi .
$$

In Fig. 3 we plot the contribution to the different components of the convergence field from a single halo $\kappa_{0 s}, \kappa_{1 s}$, and $\kappa_{2 s}$ at $z=0.3$ for $m_{\nu}=0.05 \mathrm{eV}$ in the case that the relative velocity is perpendicular to the l.o.s., i.e., $\theta=\pi / 2$. Here, we take $M=10^{13} M_{\odot}$, which is the typical mass of a halo.

Although the effect is fairly small, no other effect is known to produce the dipole term $\kappa_{1 s}$ except for the neutrino wake lensing. The halo density field itself may have a dipole; however, its contribution can be removed by correlating with the relative velocity field direction $\left\langle\delta_{h}(\boldsymbol{x}) \delta_{h}(\boldsymbol{x}+\boldsymbol{r}) \hat{\boldsymbol{r}} \cdot \hat{\boldsymbol{v}}_{\nu c}\right\rangle$, as it is antisymmetric. We have verified this is the case by $N$-body simulations; without the neutrinos the measured lensing signal is indeed consistent with zero within the numerical accuracy. The clustering of neutrinos around a growing dark matter halo which moves with a bulk relative velocity with respect to neutrinos can be calculated similarily as in Ref. [14].

Principle of observation.-We can reconstruct the relative velocity field from the large scale structure survey data $\delta_{g}$; in Fourier space the velocity is given by

$$
\boldsymbol{v}_{\nu c}(\boldsymbol{k})=\frac{i \hat{\boldsymbol{k}}}{k} \frac{\theta_{c}(k)-\theta_{\nu}(k)}{T_{c}(k)} \frac{\delta_{g}(\boldsymbol{k})}{b_{g}},
$$

where $\theta_{c}$ and $\theta_{\nu}$ are the velocity divergence transfer functions, $T_{c}$ is the density transfer function, and $b_{g}$ is a linear galaxy bias as the galaxy density field is different from that of dark matter. The influence of different values of the bias is marginal as we only need the direction of the relative velocity, which can be reconstructed accurately [15]. The lensing dipole can then be calculated with respect to the direction of the velocity, so that the neutrino wakes add coherently. However, as the neutrino mass is unknown, we would have to generate the relative velocity fields for many neutrino masses and determine the neutrino mass as the best fit for $\kappa_{1}$.

In a velocity coherent volume $V=L^{3}$, each halo produces a wake in the same direction and adds constructively to the dipole term ( $\cos \phi$ has the same value). For a simple estimate, the number of halos of typical mass in such a volume is $\bar{n} V$, where $\bar{n}=\rho_{m} / M$ and $\rho_{m}$ is the matter density at the corresponding redshift. Thus, we can multiply the response density by $\bar{n} V$, or in more sophisticated treatment calculate the integrated response using the Press-Schechter model.

There are several coherent velocity volumes along the l.o.s., and each of these contributes to the dipole term, but the dipole directions are randomly oriented with respect to one another. If the contribution to $\kappa_{1}$ from each of these coherent volumes can be measured individually, one may construct a stacked $\kappa_{1}$, which will total as the number of coherent volumes as discussed previously. However, it is too difficult to measure $\kappa_{1}$ for individual velocity coherent volumes. Instead, the total $\kappa_{1}$ along each 1.o.s. can be observed and the dipoles from each velocity coherent volume will now add incoherently, resulting that $\kappa_{1}$ grows as $\kappa_{1} \approx \kappa_{1 s} \bar{n} V \sqrt{\chi_{\text {eff }} / L}$, where $\kappa_{1 s}$ was the single halo contribution shown in Fig. 3 For our simple estimate, we define $\chi_{\text {eff }}$ as the comoving length along our l.o.s. where the lensing weight $\omega(\chi)$ is larger than half of its maximum value. For the fiducial case here, $\chi_{\mathrm{eff}}=2093.4 \mathrm{Mpc} / h$. Considering the redshift distribution of the lensing halos makes $\kappa_{1}$ about $15 \%$ smaller than this simple estimate.

Right-handed neutrinos. - If the neutrinos are Dirac type, the primordial right-handed neutrinos have the same mass as the left-handed ones, but with a different temperature, so that their distribution is also different. The decoupling of the $\nu_{R}$ 's depends on the model of their interactions, and one has to consider the rich phenomenology and experimental constraints on such models [1, 10]. Here, as a simple example, we assume that the $\nu_{R}$ 's were fully thermalized during the early Universe but decoupled before any other standard model (SM) particle. This is a plausible case, for the $\nu_{R}$ 's do not participate in the SM interactions, and their earlier thermalization must be due to a beyond the standard model interaction that freezes out at energies above $1 \mathrm{TeV}$. The temperature of these neutrinos can be computed by ensuring the conservation of entropy density $g_{* S}^{\text {today }} T_{\gamma}^{3}=g_{* S}^{\nu_{R}} T_{\nu_{R}}^{3}$, where $g_{* S}^{\nu_{R}}$ is the effective degree of freedom when the $\nu_{R}$ decoupled. If the standard model is applicable at such energy scales except for the addition of right-handed neutrinos, we have $g_{* S}^{\mathrm{SM}}=106.75$, and $g_{* S}^{\nu_{R}}=g_{* S}^{\mathrm{SM}}+\frac{7}{8}$ (fermions) $\times 3$ (number of species) $\times 2$ (particle and antiparticle). Currently, $g_{* S}=3.91$ but again a factor 
of $\frac{7}{8} \times 6 \times\left(T_{\nu_{R}} / T_{\gamma}\right)^{3}$ needs to be added. This leaves

$$
\left(\frac{T_{\nu_{R}}}{T_{\gamma}}\right)^{3}=\frac{106.75+\frac{7}{8} \times 6}{3.91+\frac{7}{8} \times 6 \times\left(\frac{T_{\nu_{R}}}{T_{\gamma}}\right)^{3}}
$$

so the extrapolated right-handed neutrino temperature is $T_{\nu_{R}}=(43 / 427)^{1 / 3} T_{\nu_{L}} \simeq 0.905 \mathrm{~K}$. With $\nu_{R}$ 's at this temperature, the number of relativistic degrees of freedom during the nucleosynthesis era is $N_{\text {eff }}=3.04 \times\left[1+\left(T_{\nu_{R}} / T_{\nu_{L}}\right)^{4}\right] \simeq 3.18$. This is consistent with the current big bang nucleosynthesis constraints [16]. Note that the $\nu_{R}$ density might be greater if its production mechanism is nonthermal, but the big bang nucleosynthesis constrains its density to be not much higher than the thermal value. To model $\nu_{R}$ 's, we use the CLASS code [17] with neutrinos of the same mass but a lowered temperature. The results presented here only depend on the $\nu_{R}$ 's being thermalized and their current temperature.

The computation of the relative velocity field of the $\nu_{R}$ is similar to the $\nu_{L}$. However, $\nu_{R}$ 's decoupled earlier with a lower temperature, i.e., velocity dispersion, so the relative velocity is different from that of the $\nu_{L}$. The $\nu_{L}$ and $\nu_{R}$ relative velocity spectra are shown in Fig. 11. The coherent scale is $R=10.0 \mathrm{Mpc} / h$ for the $\nu_{R}$ at $z=0.3$. In this model we take $\sqrt{\left\langle v_{\nu_{R} c}^{2}\right\rangle}=373 \mathrm{~km} / \mathrm{s}$ as the typical relative velocity. The one-dimensional neutrino velocity dispersion is $\sigma(z)=2.077 T_{\nu}(z) / m_{\nu} \simeq 1263 \mathrm{~km} / \mathrm{s}$ for the $\nu_{R}$ at $z=0.3$. The direction of the flow deviates from that of the $\nu_{L}$, with an angle $\langle\cos \theta\rangle=$ $\int \Delta_{\zeta}^{2} \theta_{\nu c}^{L} \theta_{\nu c}^{R} / k^{3} d k / \sqrt{\int \Delta_{\zeta}^{2}\left(\theta_{\nu c}^{L} / k\right)^{2} d \ln k \int \Delta_{\zeta}^{2}\left(\theta_{\nu c}^{R} / k\right)^{2} d \ln k}$, and we find $\theta \sim 20^{\circ}$.

The right-handed neutrino wake direction differs from the left-handed wake by an angle of typically $20 \mathrm{deg}$. This direction is computable, and differs from patch to patch. The signal is a small effect on the amplitude of the total wake, which is degenerate with measurement systematics and small variations in the left-handed neutrino mass. Instead, we use the difference in direction, and only consider the right-handed wake component orthogonal to the left-handed wake to be observable, and adjust the sensitivity estimates correspondingly.

The observability.-For forecasting purposes, we consider a combined LSST+Euclid data set as well as a future $21 \mathrm{~cm}$ lensing survey. The LSST will survey about three billion galaxies and the expected error for $\kappa$ is about $0.28 / \sqrt{N} \simeq$ $5.2 \times 10^{-6}$, where $N=2.88 \times 10^{9}[13]$. The Euclid survey gives a similar expected error [18] 19]. Here, for simple estimates we will neglect the overlap between these two surveys and assume that they provide independent data sets. By combining these data, we can reach a precision of $\sigma_{\kappa_{1}}=5.2 \times 10^{-6} / \sqrt{2}=3.68 \times 10^{-6}$.

Ultimately, extremely high precision measurements can be achieved with $21 \mathrm{~cm}$ lensing surveys such as the one proposed in Ref. [20]. For such a survey, the error on $\kappa$ is $\left(k_{\max } / k_{\min }\right)^{-1.5}$. For a survey with $k_{\max }=1.47 \times$ $10^{2} \mathrm{~h} / \mathrm{Mpc}$ and $k_{\min }=1.47 \times 10^{-3} \mathrm{~h} / \mathrm{Mpc}$, the expected error is about $3.16 \times 10^{-8}$, which is far smaller than the sig-

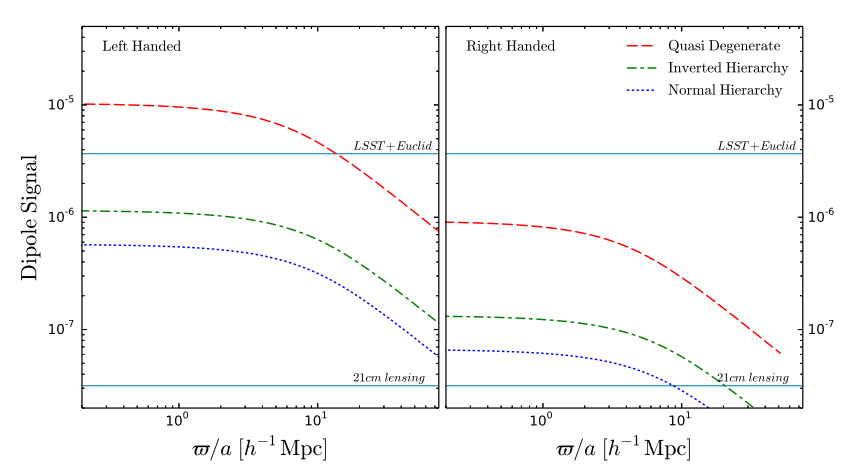

FIG. 4: Dipole signal $\bar{\kappa}_{1}$ with consideration of neutrino hierarchy. The noise floors for LSST+Euclid lensing and for $21 \mathrm{~cm}$ lensing are also shown. For the right panel, only the signal orthogonal to the left-handed neutrino wake is shown.

nals. With such future $21 \mathrm{~cm}$ lensing surveys, we can measure neutrino masses with unprecedented precision, and may even determine whether the neutrinos are Majorana or Dirac particles.

Based on the measurement of neutrino mass differences from the neutrino oscillation experiments, three possible hierarchies are being considered: (i) the normal hierarchy $(\mathrm{NH})$ case, with $m_{1} \sim m_{2} \approx 0, m_{3} \approx 0.05 \mathrm{eV}$; (ii) the inverted hierarchy (IH) case, with $0 \sim m_{3} \ll m_{1} \sim m_{2} \approx 0.05 \mathrm{eV}$; (iii) the quasidegenerate (QD) case, with $m_{1} \approx m_{2} \approx m_{3} \approx$ $0.1 \mathrm{eV}$. In the IH and NH cases, we can neglect the contribution of the lighter neutrinos, so the $\mathrm{NH}$ case is equivalent to a single neutrino with a mass of $0.05 \mathrm{eV}$, while the $\mathrm{IH}$ case is equivalent to two $0.05 \mathrm{eV}$ neutrinos. The QD case is almost equivalent to three $0.1 \mathrm{eV}$ neutrinos.

The dipoles on the sky depend on the angle $\theta$ between the relative velocity direction and the l.o.s.; the signal reaches its maximum when the relative velocity is perpendicular to the 1.o.s. $(\theta=\pi / 2)$ and vanishes when $\theta=0$. Thus, the final observed signal strength is an average over different angles. In Fig. 4, we plot the expected dipole term $\bar{\kappa}_{1}=\int \kappa_{1}(\theta) d \theta / \int d \theta$ for the $\nu_{L}$ (left panel) and the $\nu_{R}$ (right panel) for these three cases. The dipole signal for the $\nu_{R}$ is suppressed by $\cos 20^{\circ}$ since we only consider the wake component geometrically orthogonal to the $\nu_{L}$. We also plot the measurement errors for the LSST+Euclid lensing survey and the future $21 \mathrm{~cm}$ lensing survey described above. The LSST+Euclid data should be able to positively detect the QD case. With the future $21 \mathrm{~cm}$ lensing survey, the $\mathrm{NH}$ and $\mathrm{IH}$ cases can be distinguished, and even the $\nu_{R}$ may be detectable.

Conclusion.-We have computed the density contrast of neutrino wakes produced by the relative motions of neutrinos and dark matter. We have estimated the observability of these wakes via gravitational lensing and have shown that it may be possible to observe both the mass hierarchy as well as righthanded neutrinos. The wake directions differ by a distinctive angular signature.

We acknowledge the support of the Chinese MoST 863 program under Grant No. 2012AA121701, the CAS Sci- 
ence Strategic Priority Research Program XDB09000000, the NSFC under Grant No. 11373030, Tsinghua University, CHEP at Peking University, and NSERC.

[1] K. A. Olive and Particle Data Group, Chinese Physics C 38, 090001 (2014).

[2] R. N. Cahn, D. A. Dwyer, S. J. Freedman, W. C. Haxton, R. W. Kadel, Y. G. Kolomensky, K. B. Luk, P. McDonald, G. D. Orebi Gann, and A. W. P. Poon, ArXiv e-prints (2013), 1307.5487.

[3] J. R. Bond, G. Efstathiou, and J. Silk, Physical Review Letters 45, 1980 (1980).

[4] W. Hu, D. J. Eisenstein, and M. Tegmark, Physical Review Letters 80, 5255 (1998), astro-ph/9712057.

[5] S. Dodelson and J. Lykken, ArXiv e-prints (2014), 1403.5173.

[6] H.-M. Zhu, U.-L. Pen, X. Chen, D. Inman, and Y. Yu, Physical Review Letters 113, 131301 (2014), 1311.3422.

[7] A. de Gouvea, K. Pitts, K. Scholberg, G. P. Zeller, J. Alonso, A. Bernstein, M. Bishai, S. Elliott, K. Heeger, K. Hoffman, et al., ArXiv e-prints (2013), 1310.4340.

[8] S. Betts, W. R. Blanchard, R. H. Carnevale, C. Chang, C. Chen, S. Chidzik, L. Ciebiera, P. Cloessner, A. Cocco, A. Cohen, et al., ArXiv e-prints (2013), 1307.4738.

[9] A. J. Long, C. Lunardini, and E. Sabancilar, J. Cosmology Astropart. Phys. 8, 038 (2014), 1405.7654.
[10] P. Langacker, Reviews of Modern Physics 81, 1199 (2009), 0801.1345 .

[11] Z.-Z. Xing, International Journal of Modern Physics A 23, 4255 (2008), 0810.1421.

[12] J. Binney and S. Tremaine, Galactic Dynamics: Second Edition (Princeton University Press, 2008).

[13] LSST Science Collaboration, P. A. Abell, J. Allison, S. F. Anderson, J. R. Andrew, J. R. P. Angel, L. Armus, D. Arnett, S. J. Asztalos, T. S. Axelrod, et al., ArXiv e-prints (2009), 0912.0201.

[14] M. LoVerde and M. Zaldarriaga, Phys. Rev. D 89, 063502 (2014), 1310.6459.

[15] D. Inman, J. D. Emberson, U.-L. Pen, A. Farchi, H.-R. Yu, and J. Harnois-Déraps, Phys. Rev. D 92, 023502 (2015), 1503.07480.

[16] L. A. Anchordoqui, H. Goldberg, and G. Steigman, Physics Letters B 718, 1162 (2013), 1211.0186.

[17] D. Blas, J. Lesgourgues, and T. Tram, J. Cosmology Astropart. Phys. 7, 034 (2011), 1104.2933.

[18] R. Laureijs, J. Amiaux, S. Arduini, J. . Auguères, J. Brinchmann, R. Cole, M. Cropper, C. Dabin, L. Duvet, A. Ealet, et al., ArXiv e-prints (2011), 1110.3193.

[19] L. Amendola, S. Appleby, D. Bacon, T. Baker, M. Baldi, N. Bartolo, A. Blanchard, C. Bonvin, S. Borgani, E. Branchini, et al., Living Reviews in Relativity 16, 6 (2013), 1206.1225.

[20] K. W. Masui and U.-L. Pen, Physical Review Letters 105, 161302 (2010), 1006.4181. 